\title{
Interactions of Polyethylenimines with Zwitterionic and
}

\section{Anionic Lipid Membranes}

Urszula Kwolek, Dorota Jamróz, Małgorzata Janiczek, Maria Nowakowska, Paweł Wydro, Mariusz

Kepczynski

Faculty of Chemistry, Jagiellonian University, Ingardena 3, 30-060 Kraków, Poland

\section{Monolayer Experiments}

Table s1. Area per Lipid $(A)$ at $\pi=30 \mathrm{mN} / \mathrm{m}$.

\begin{tabular}{cccc}
\hline & \multicolumn{3}{c}{$A\left(\mathrm{~nm}^{2}\right)$} \\
\cline { 2 - 4 }$C_{\text {PEI }}(\mu \mathrm{g} / \mathrm{mL})$ & POPC & DOPA & POPC/DOPA (9:1) \\
\hline lPEI & 0.644 & 0.590 & 0.636 \\
6 & 0.647 & 0.638 & 0.651 \\
60 & 0.653 & 0.676 & 0.679 \\
bPEI & & & 0.636 \\
0 & 0.644 & 0.590 & 0.655 \\
6 & 0.647 & 0.623 & 0.675 \\
\hline 6 & 0.654 & 0.638 & \\
\hline
\end{tabular}




\section{DLS Measurements}

Table s2. Values of the Mean Hydrodynamic Diameter (dz), Polydispersity (PDI) and Zeta Potential $(\zeta)$ of POPC SUVs Dispersed in a $1 \mathrm{mM} \mathrm{NaCl} \mathrm{Solution} \mathrm{at} \mathrm{pH} 7.4$ and Treated with PEIs (Values Are the Mean \pm Standard Deviation).

\begin{tabular}{lllllc}
\hline system & $\begin{array}{l}\text { polycation } \\
\text { concentration } \\
(\mu \mathrm{g} / \mathrm{mL})\end{array}$ & $\begin{array}{l}\text { polycation } \\
\text { content } \\
(\mathrm{wt} \%)\end{array}$ & $\mathrm{dz}(\mathrm{nm})$ & PDI & $\zeta(\mathrm{mV})$ \\
& 0 & 0 & $113.4 \pm 0.8$ & $0.07 \pm 0.02$ & $-11.9 \pm 0.6$ \\
& 20 & 2.0 & $121.3 \pm 1.3$ & $0.22 \pm 0.02$ & $-10.4 \pm 0.1$ \\
POPC SUVs & 60 & 6.4 & $>10^{3}$ & $1.00 \pm 0.00$ & $-7.5 \pm 0.2$ \\
POPC SUVs & 100 & 11.1 & $>10^{3}$ & $1.00 \pm 0.00$ & $-4.9 \pm 0.1$ \\
with IPEI & 160 & 19.0 & $>10^{3}$ & $1.00 \pm 0.00$ & $-3.0 \pm 0.2$ \\
& 0 & 0 & $115.2 \pm 2.3$ & $0.26 \pm 0.01$ & $-4.2 \pm 0.2$ \\
POPC SUVs & 100 & 11.1 & $157.0 \pm 11.9$ & $0.56 \pm 0.03$ & $1.60 \pm 0.04$ \\
POPC SUVs & 150 & 17.6 & $195.8 \pm 14.1$ & $0.65 \pm 0.03$ & $2.15 \pm 0.05$ \\
with bPEI & 200 & 25.0 & $377.2 \pm 44.6$ & $0.46 \pm 0.02$ & $2.7 \pm 0.2$ \\
& 250 & 33.3 & $247.7 \pm 16.7$ & $0.41 \pm 0.02$ & $4.3 \pm 0.2$ \\
\hline
\end{tabular}

\section{Simulations}

Initial Configurations of PEI-Membrane Systems. 

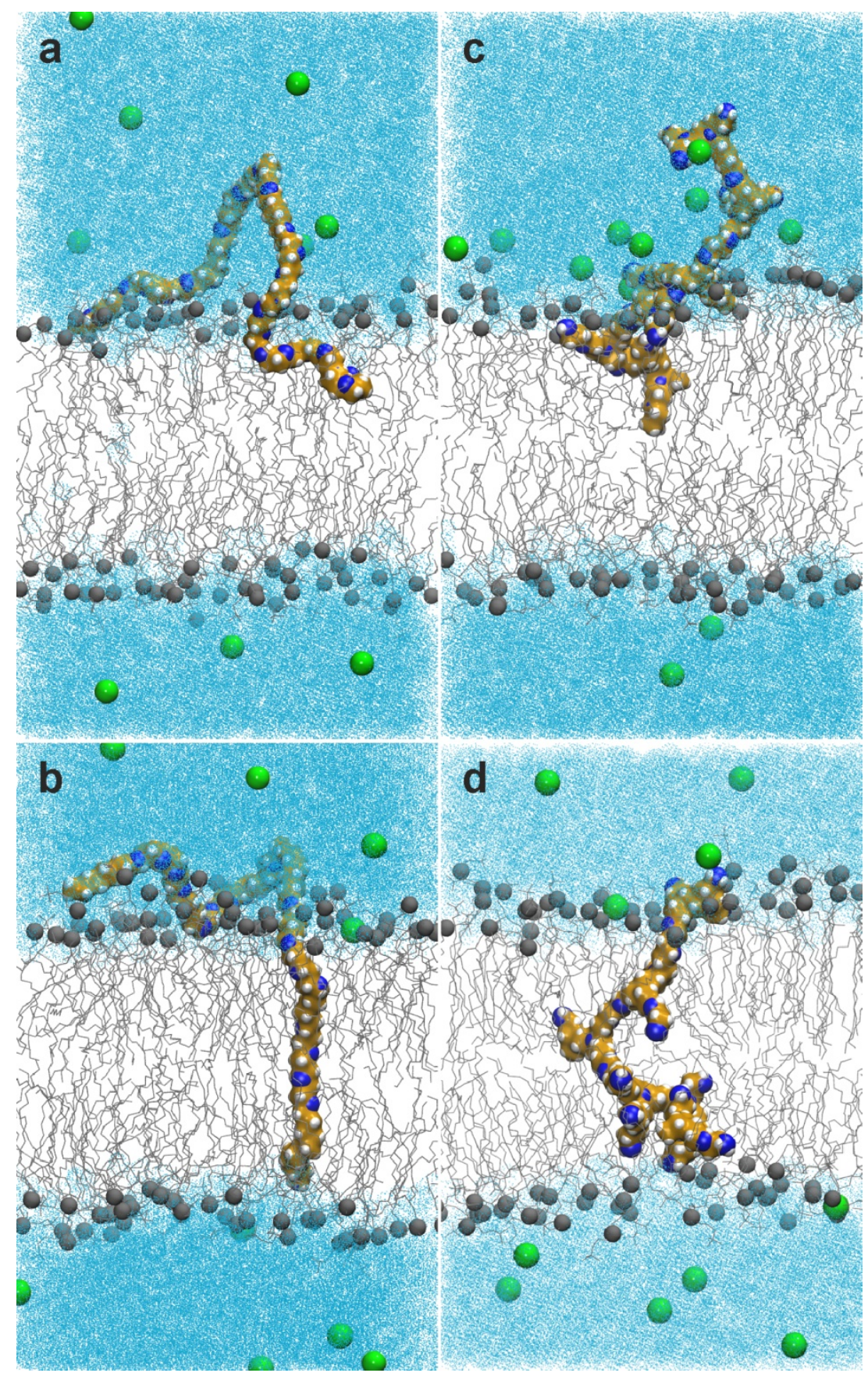

Figure s1. Initial configurations of PEI-POPC systems: S1-IPEI (a), E1-lPEI (b), S1-bPEI (c), and E1bPEI (d). PEI molecules are drawn as van der Waals spheres (C, orange; N, blue; H, white). Lipids are shown as gray sticks, and their phosphate groups are shown as gray spheres. $\mathrm{Cl}^{-}$anions are represented by green spheres. 


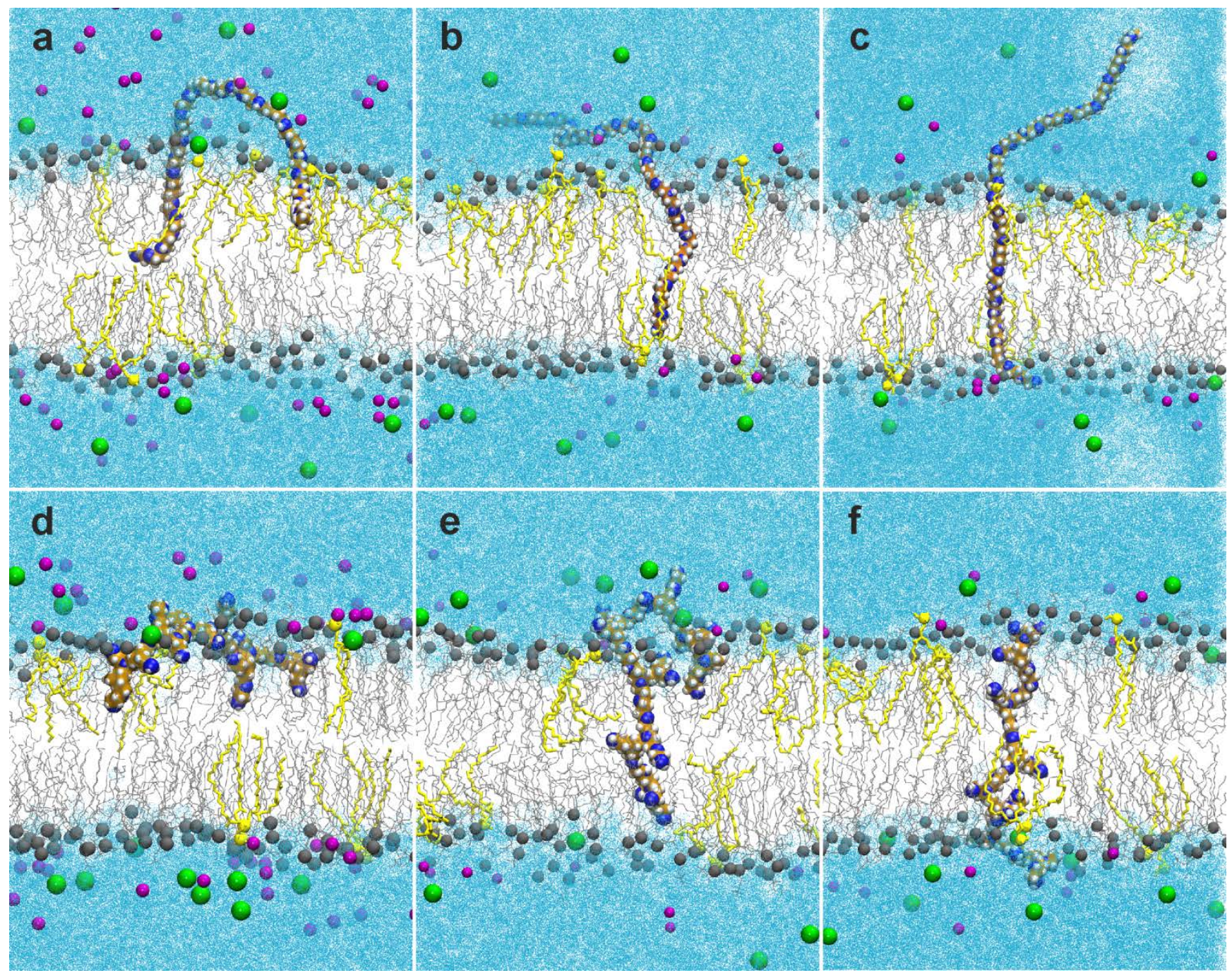

Figure s2. Initial configurations of PEI-POPC/DOPA systems: S3-lPEI (a), E2-IPEI (b), T2-lPEI (c), S3-bPEI (d), E2-bPEI (e), and T2-bPEI (f). PEI molecules are drawn as van der Waals spheres (C, orange; $\mathrm{N}$, blue; $\mathrm{H}$, white). DOPA molecules are shown as yellow sticks with yellow spheres for the phosphate groups. POPC molecules are shown as gray sticks and their phosphate groups are shown as gray spheres. $\mathrm{Cl}^{-}$and $\mathrm{K}^{+}$ions are represented by green and violet spheres, respectively.

\section{Conformation of PEIs in Water and Hydration of Polymer Chains.}

Initial configurations, generated as structures optimized in vacuum conditions (Figures s3a and b), were put into water boxes with ca. 9000 water molecules and subjected to a short simulation of 10 ns. The final configurations after these short simulations (Figures s3c and d) reveal that both PEIs exhibit 
strong affinity to water, which results in rapid hydration of the polymer with simultaneous release of the originally tightly bound counterions and stretching out the oligomer main chains due to repulsion between the charged amine units. The systems of PEIs in the water boxes were next equilibrated for additional 50 ns to determine the hydration number of the polymer units (Figure s4).
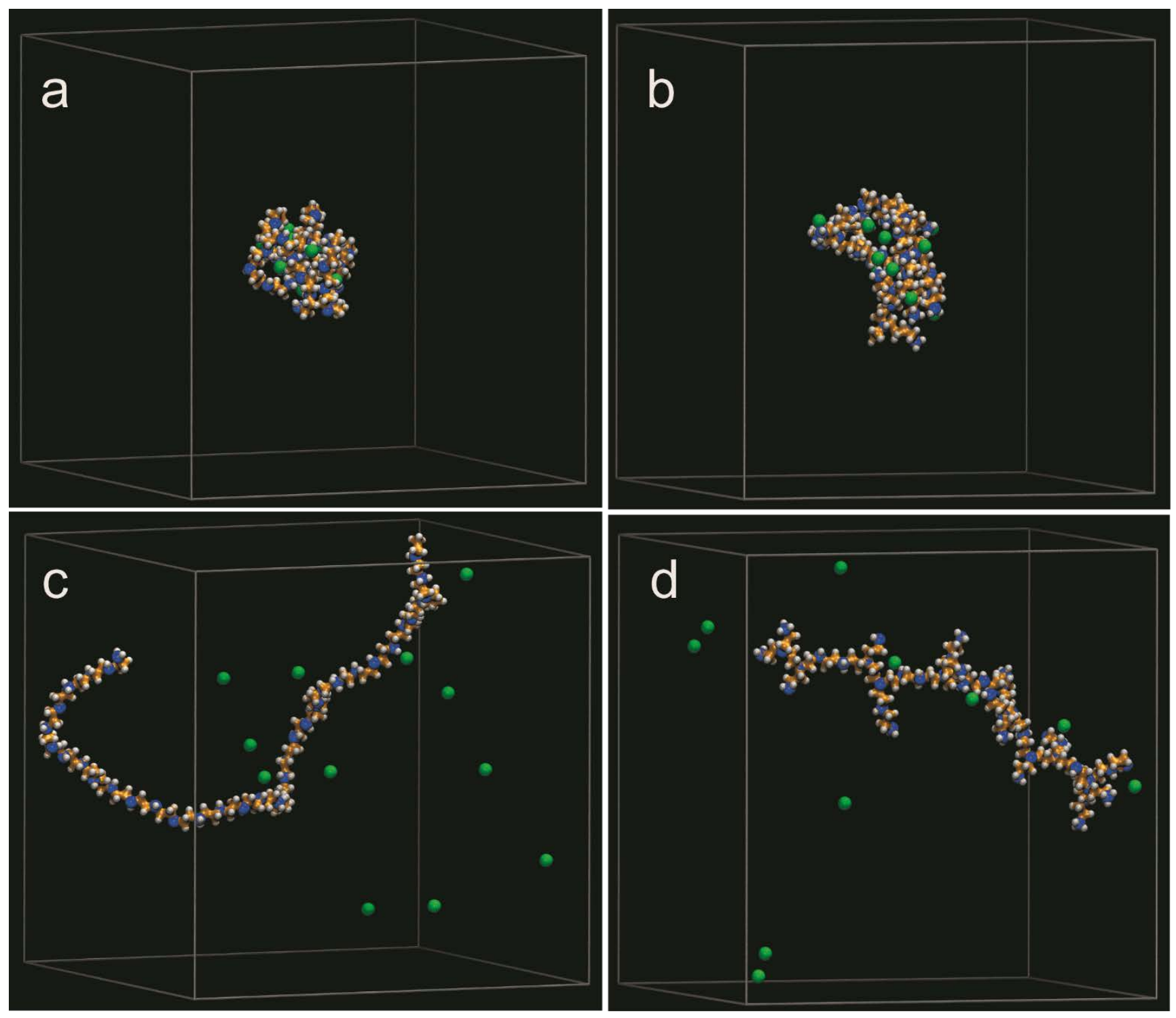

Figure s3. Initial configurations of IPEI (a) and bPEI (b) and configurations of IPEI (c) and bPEI (d) after 10 ns simulations in water. PEI molecules are drawn as van der Waals surfaces (C, orange; N, blue; $\mathrm{H}$, white). $\mathrm{Cl}^{-}$anions are represented by green spheres. Water molecules are not shown for clarity. 

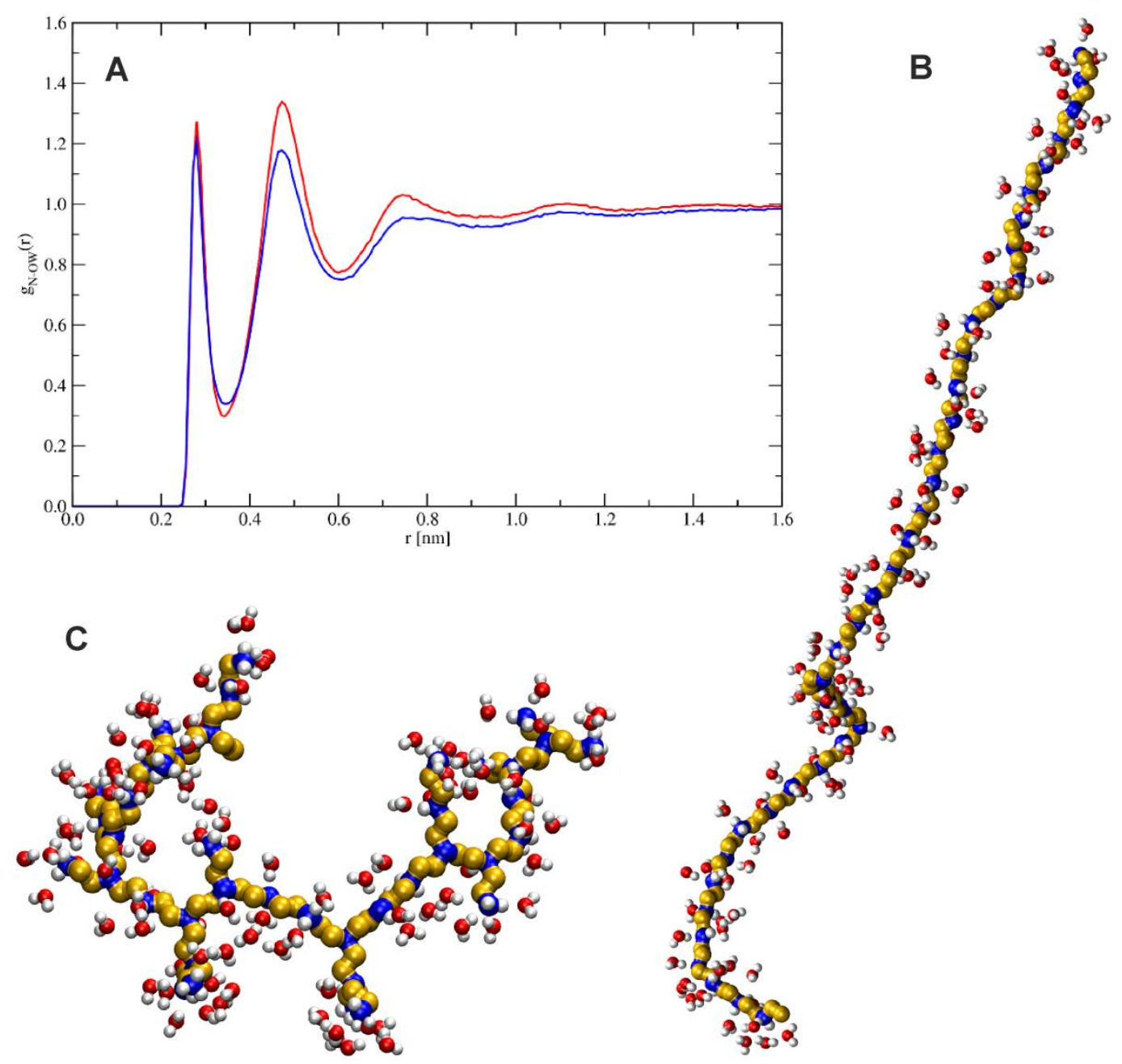

Figure s4. (A) The $g_{\mathrm{N}-\mathrm{OW}}(r)$ radial distribution functions for the atomic pairs of PEI nitrogens and $\mathrm{O}$ of water for IPEI (red) and bPEI (blue). The functions were averaged over the last $30 \mathrm{~ns}$ of the trajectories. Snapshots of 40-mers of IPEI (B) and bPEI (C) taken at the end of the $50 \mathrm{~ns}$ simulations. PEI chains are shown in a licorice representation in yellow for carbon atoms, in blue for $\mathrm{N}$ atoms, and white for $\mathrm{H}$ atoms. Water molecules in the first hydration (the $\mathrm{N}-\mathrm{O}$ distance $\leq 0.35 \mathrm{~nm}$ ) shell are shown as sticks and colored as follows: red, oxygens and white, hydrogens. 

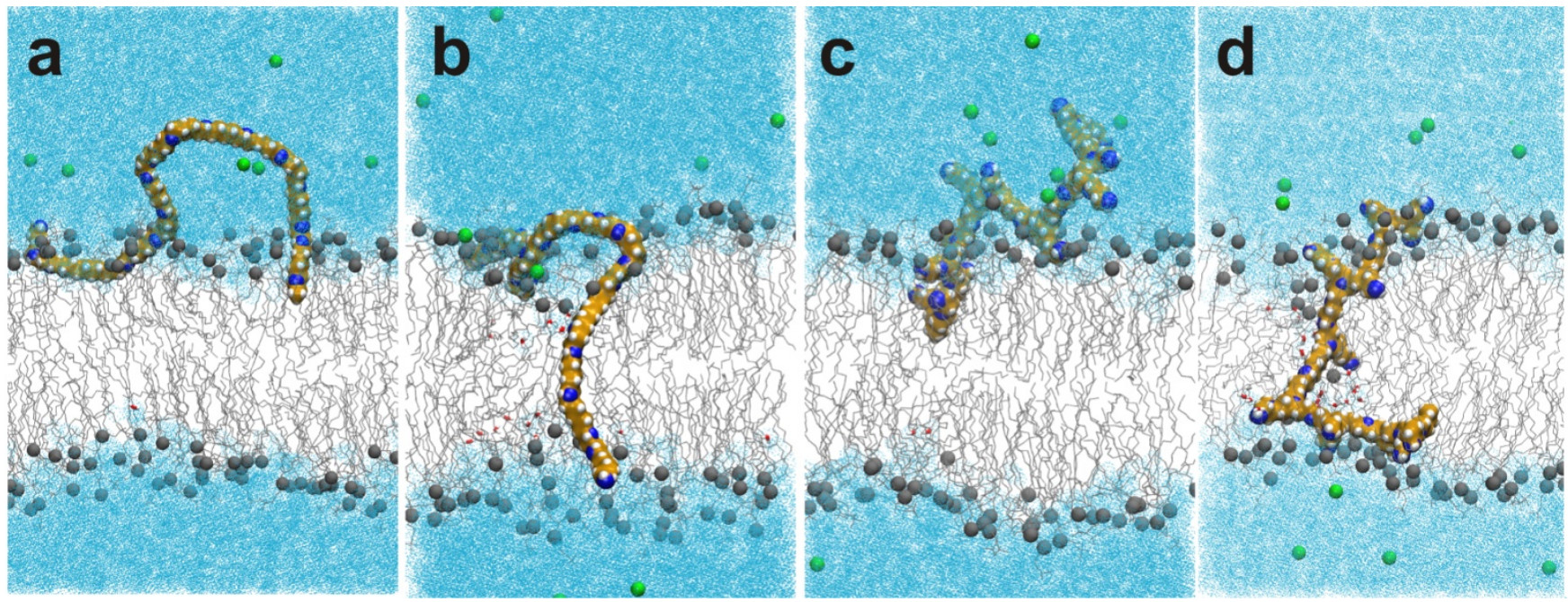

Figure s5. Configurations of PEI-POPC systems after 200 ns simulations: S1-IPEI (a), E1-IPEI (b), S1bPEI (c), E1-bPEI (d). PEI molecules are drawn as van der Waals surfaces (C, orange; N, blue; H, white). Lipids are shown as gray sticks, and their phosphate groups are shown as gray spheres. $\mathrm{Cl}^{-}$ anions are represented by green spheres. For the sake of clarity only a slice of the membrane containing the polymer is shown. 

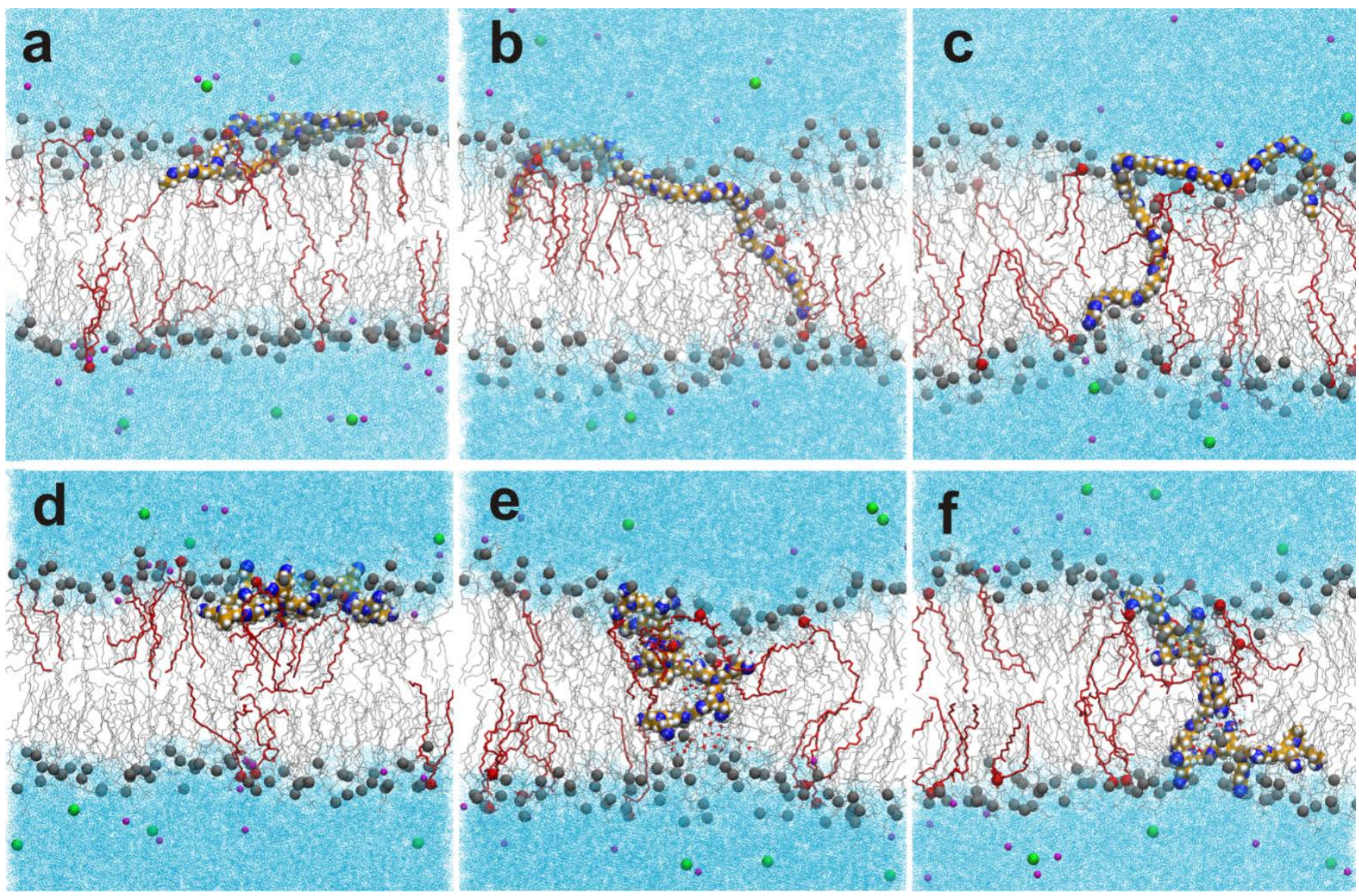

Figure s6. Configurations of PEI-POPC/DOPA systems after 200 ns simulations: : S3-IPEI (a), E2-IPEI

(b), T2-IPEI (c), S3-bPEI (d), E2-bPEI (e), and T2-bPEI (f). PEI molecules are drawn as van der Waals surfaces (C, orange; $\mathrm{N}$, blue; $\mathrm{H}$, white). DOPA molecules are shown as yellow sticks with yellow spheres for the phosphate groups. POPC molecules are shown as gray sticks, and their phosphate groups are shown as gray spheres. $\mathrm{Cl}^{-}$and $\mathrm{K}^{+}$ions are represented by green and violet spheres, respectively. For the sake of clarity only a slice of the membrane containing the polymer is shown.

\section{Calculation of H-Bonds.}

An $\mathrm{H}$-bond was assumed to be formed when the distance between the hydrogen donor and acceptor was $\leq 0.35 \mathrm{~nm}$ and deviation of the $\mathrm{D}-\mathrm{H} \cdots \mathrm{A}$ angle from the straight line was $\leq 30^{\circ}$. 


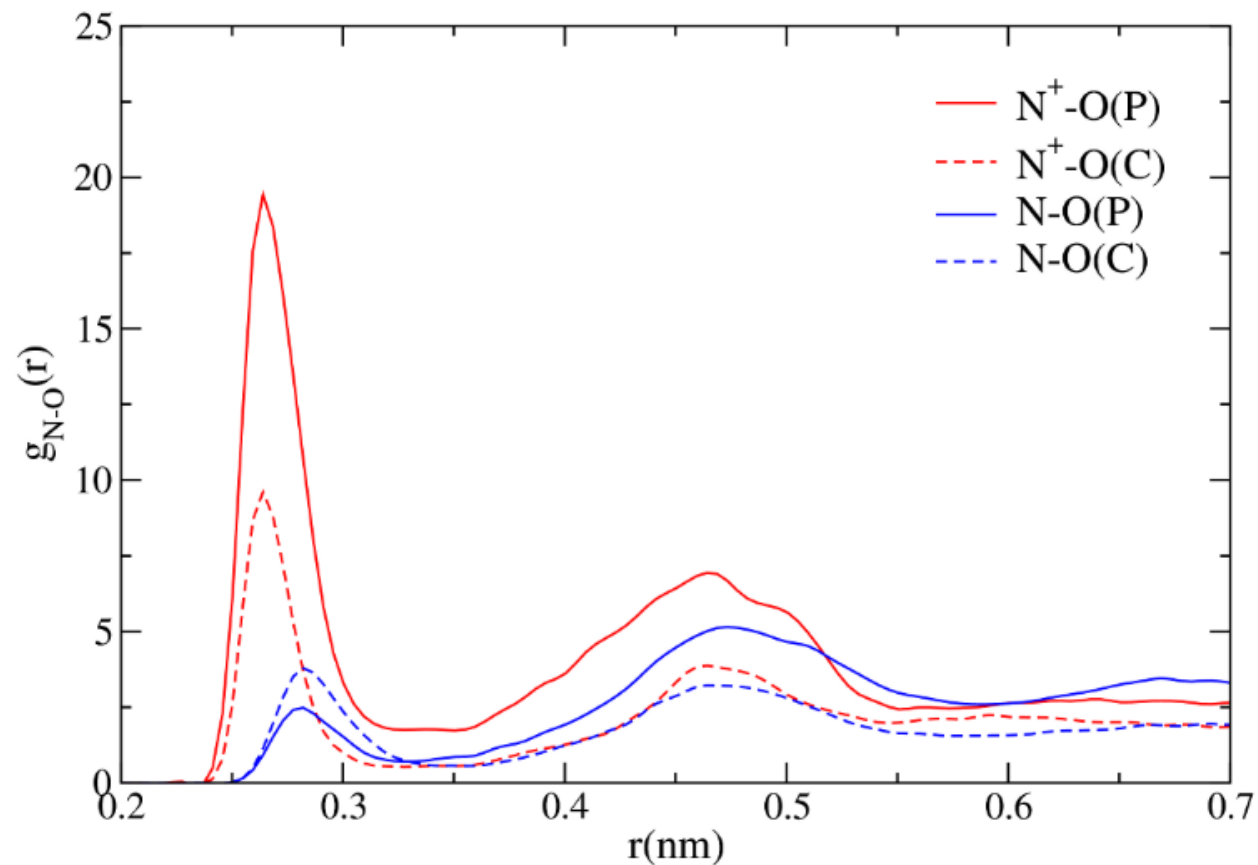

Figure s7. (A) The $g_{\mathrm{N}-\mathrm{O}}(r)$ radial distribution functions for the atomic pairs of IPEI nitrogen atoms and the lipids oxygen atoms of the phosphate $(\mathrm{O}(-\mathrm{P}))$ and the ester $(\mathrm{O}-(\mathrm{C}))$ groups. 

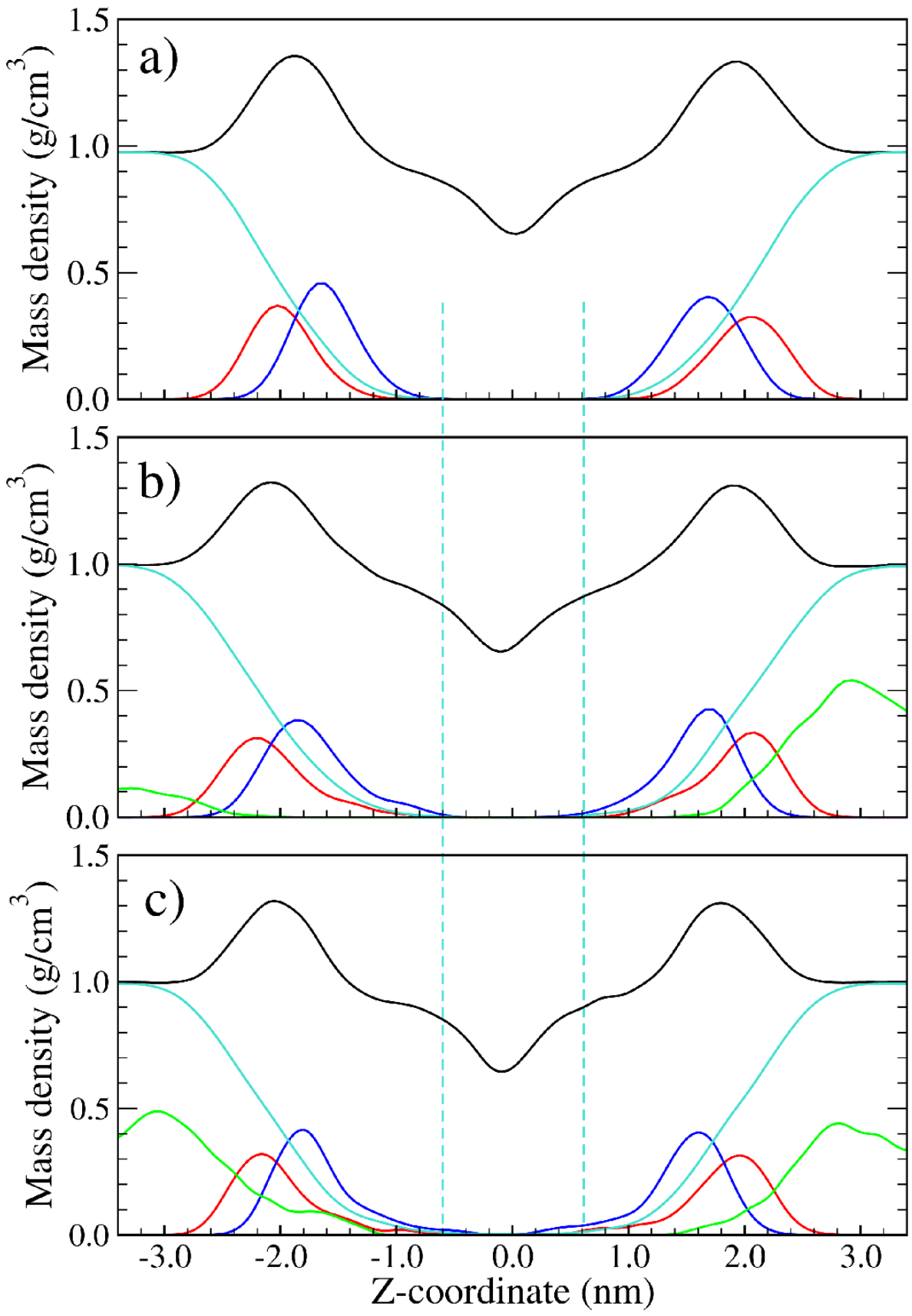

Figure s8. Mass density profiles of the headgroup atoms, water, and $\mathrm{Cl}^{-}$anions across the POPC membrane (a) and the membranes with fully immersed IPEI (system E1-lPEI) (b) and bPEI (system E1bPEI) (c). The range of the hydrophobic core was marked with cyan dashed lines. The density profiles were averaged over the last 50 ns for the pure membrane and 100 ns for the membrane/PEI systems. The profiles are colored as follows: black lines, overall system density; cyan lines, water; blue lines, POPC glycerol; red lines, POPC phosphate; light green lines, $\mathrm{Cl}^{-}$anions. The $\mathrm{Cl}^{-}$profiles were multiplied by a factor of 100 . 


\section{POPC/DOPA membranes}

We simulated the POPC membranes doped with $\sim 10$ mol\% of $\mathrm{DOPA}^{-}$or $\mathrm{DOPA}^{2-}$. Figure s9a shows mass density profiles for the hydrophilic part in the POPC/DOPA ${ }^{-}$membrane. Location of maxima for the phosphate groups is within $0.1 \mathrm{~nm}$ the same for POPC and DOPA- showing that, in spite of different sizes of the headgroups in both lipids, their phosphate groups are well aligned. Thickness of both POPC/DOPA membranes (calculated as the distance between the maxima for the phosphate groups in both leaflets) equal $4.0 \mathrm{~nm}$, which corresponds very well to the MD result for similar 1,2-dioleoyl-snglycero-3-phosphocholine (DOPC)/DOPA (9:1) bilayer of smaller size (128 lipids) simulated by Kooijman et al. ${ }^{1}$ Also, the calculated area per lipid was the same for both membranes $0.57 \pm 0.01 \mathrm{~nm}^{2}$, which is very close to the measured value for the pure POPC bilayer $\left(0.59 \mathrm{~nm}^{2}\right){ }^{2}$ 

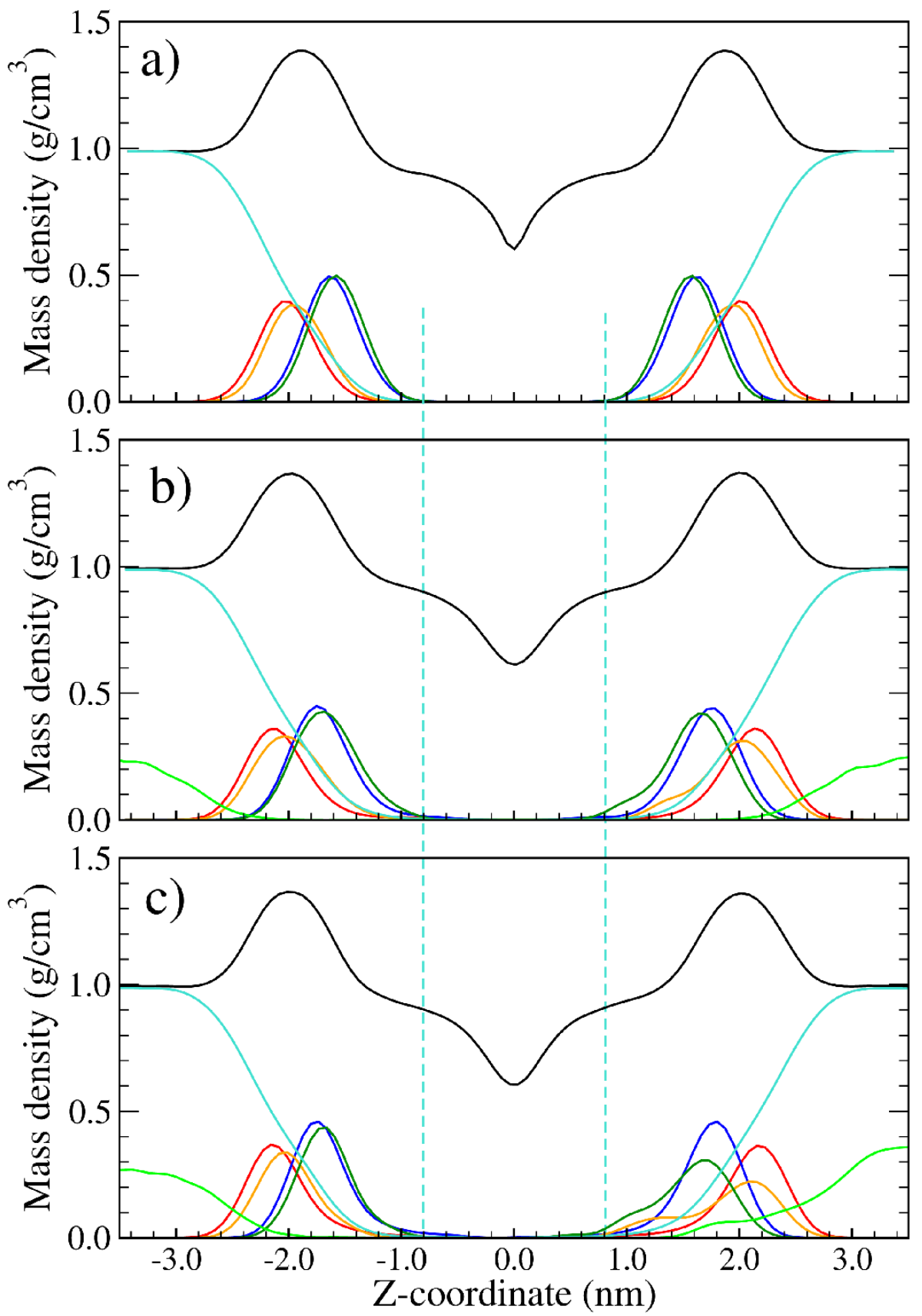

Figure s9. Mass density profiles of the headgroup atoms, water, and $\mathrm{Cl}^{-}$anions across the POPC/DOPA ${ }^{-}$membrane (a) and the membranes with fully immersed IPEI (system E2-lPEI) (b) and bPEI (system E2-bPEI) (c). The range of the hydrophobic core was marked with cyan dashed lines. The density profiles were averaged over the last 50 ns for the pure membrane and 100 ns for the membranePEI systems. The profiles are colored as follows: black lines, overall system density; cyan lines, water; blue lines, POPC glycerol; red lines, POPC phosphate; green lines, DOPA glycerol; orange lines, DOPA phosphate; light green lines, $\mathrm{Cl}^{-}$anions. The DOPA and $\mathrm{Cl}^{-}$profiles were multiplied by factors of 6 and 100, respectively. 


\section{Diffusion coefficient calculations}

The lateral and normal diffusion coefficients, $D_{\mathrm{L}}$ and $D_{\mathrm{N}}$, were computed by means of the Einstein's relation

$$
D=\lim _{t \rightarrow \infty} \frac{\operatorname{MSD}(t)}{2 d t}
$$

where $d$ is the number of translational degrees of freedom (here $d=2$ for $D_{\mathrm{L}}$ and $d=1$ for $D_{\mathrm{N}}$ ) and $\operatorname{MSD}(t)$ is the mean-squared displacement

$$
\operatorname{MSD}(t)=\left\langle|r(\tau+t)-r(\tau)|^{2}\right\rangle
$$

where $r(\tau)$ is the initial position of the center of mass and $\langle\ldots\rangle$ denotes averaging over different initial times $\tau$ during a simulation run and also over all the considered molecules in the system.

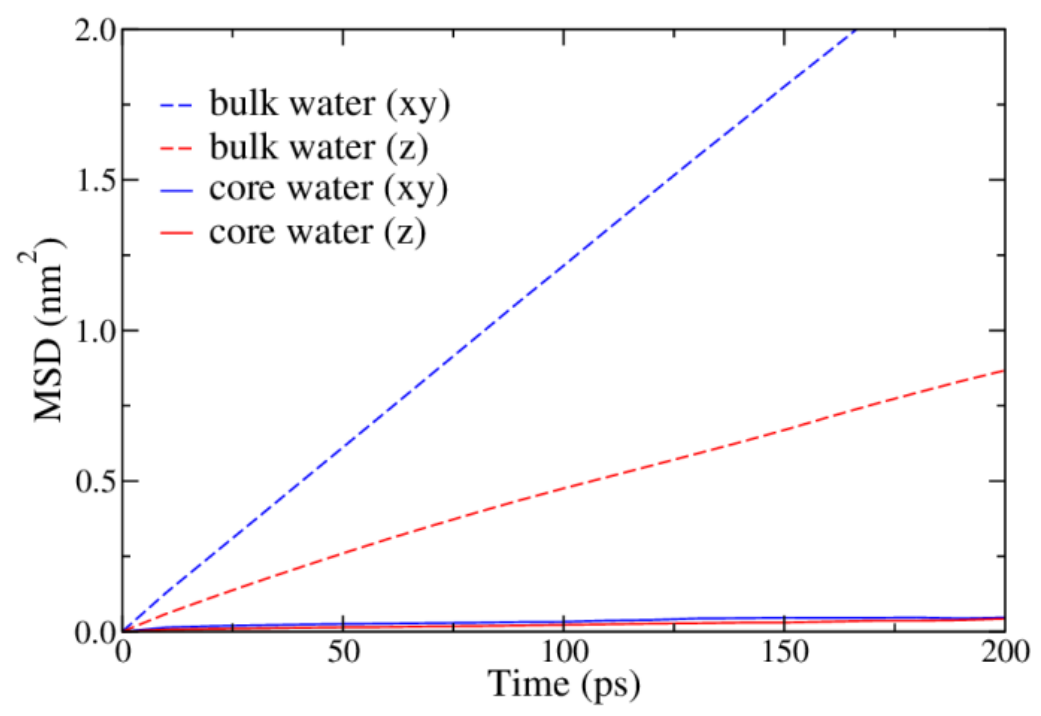

Figure s10. Mean square displacement (MSD) of “core” water (solid lines) and "bulk” water (dashed lines) in the normal (red lines) and lateral (blue lines) directions calculated for system E2-bPEI. 


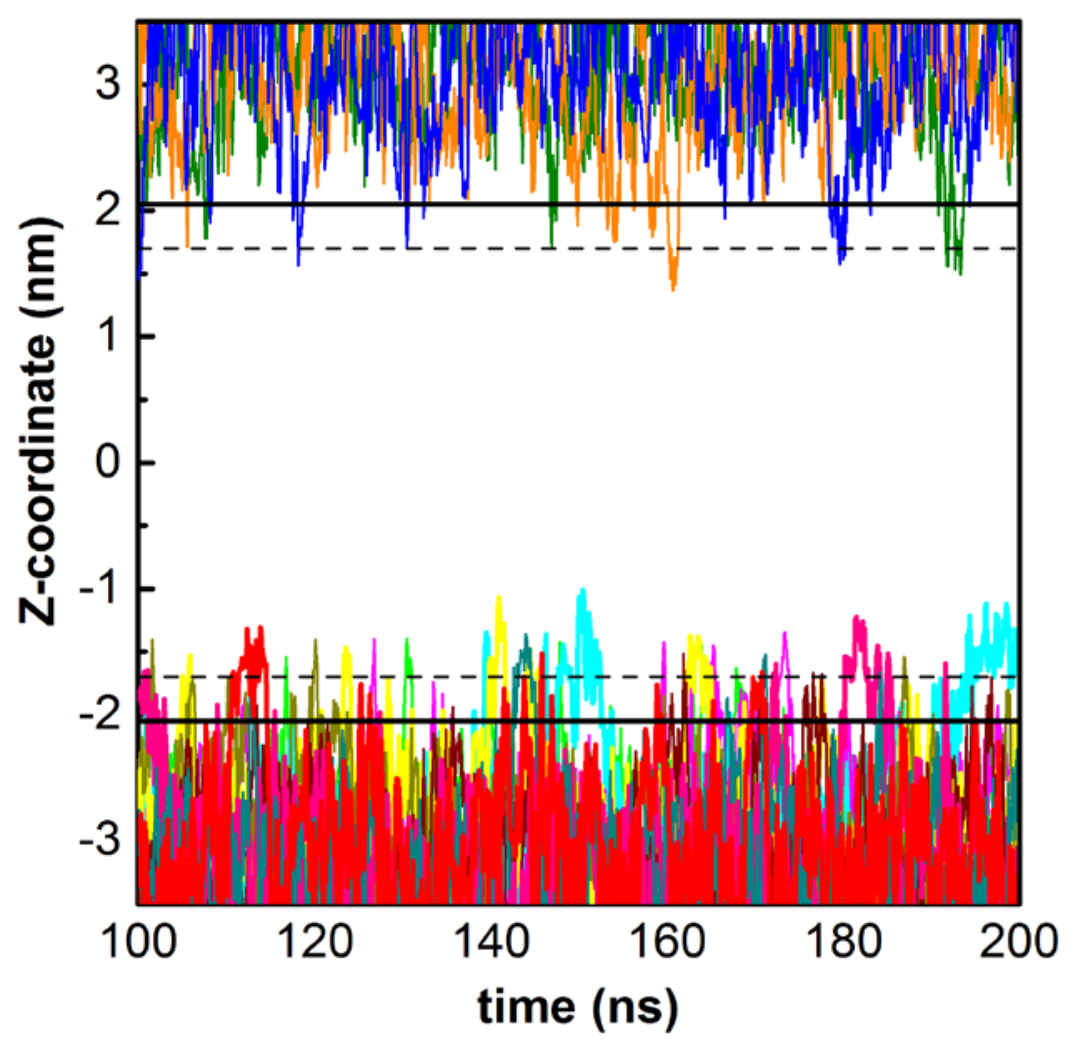

Figure s11. Trajectory of the Z coordinate (along the bilayer normal) of the chloride anions (various colors) for system E1-bPEI. The interfaces of the bilayer-water and polar-hydrophobic regions are shown as solid and dashed black lines, respectively, describing the average positions of the lipid phosphates and the glycerol groups. 

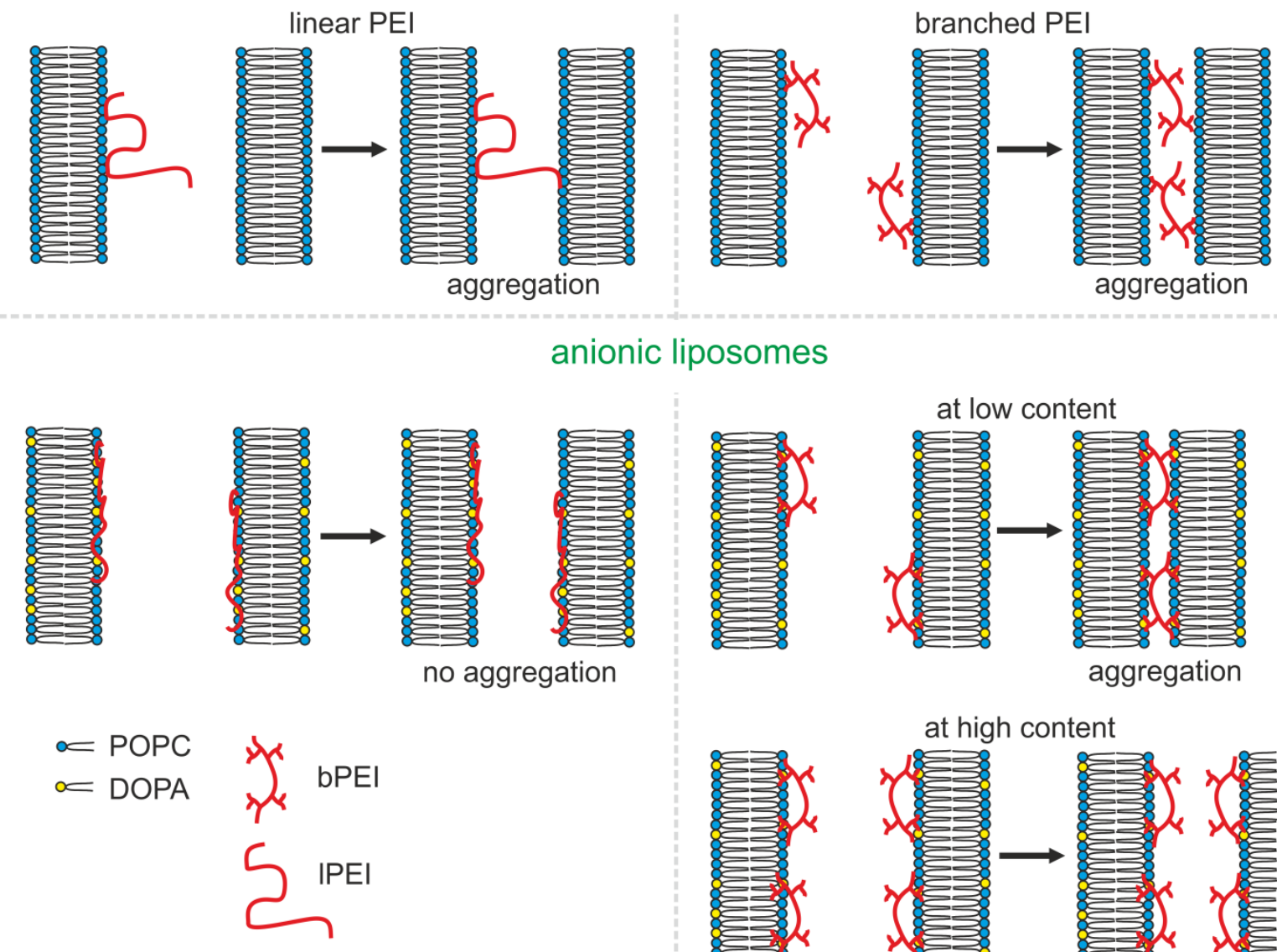

\section{liposomes}

